\title{
EL SILENCIO DEL ART BRUT: UNA VISIÓN DIFERENTE DE UN ARTE CONECTADA A LA VIDA
}

\section{EDUCATIONAL RELATION FROM THE TEACHER PERFORMANCE ETHICS PERSPECTIVE}

\author{
Cecilia Mateo Sánchez: UCAM Universidad Católica San Antonio de Murcia. \\ España. \\ cmateo@ucam.edu
}

Isabel Mengual Luna: UCAM Universidad Católica San Antonio de Murcia. España.

\section{RESUMEN}

Durante los últimos 60 años, el panorama artístico ha sufrido más cambios que a lo largo de toda su historia. Las vanguardias de la Modernidad dejaron claro testimonio de ruptura respecto al arte predecesor, tanto en su forma como en su contenido. Al final de la Modernidad todavía podía hablarse de disciplinas como pintura, escultura, grabado o dibujo, aun con sentido, desde hace 60 años se habla de otras prácticas tales como, instalación, performance, body art, land art, video art o art brut, etc., sin que estas puedan ser incluidas en los grandes géneros de las artes conocidos por todos. La enorme cantidad de innovaciones ocurridas en el campo del arte desde la Segunda Guerra Mundial nos obliga por lo tanto, a denominar este nuevo periodo de una forma distinta al de modernidad, denominándolo entonces: postmodernidad. Es en este contexto, donde situamos el arte bruto o art brut, arte marginal u outsider art y la independencia que estas prácticas poseen de lo que actualmente se entiende por aprendizaje artístico. Surge así, la necesidad de una nueva crítica y una nueva historiografía para entender, analizar y juzgar, estas nuevas formas y mecanismos de producir, que nos conduzcan al entendimiento del arte postmoderno. Nos plantearemos entonces si hay detrás de ellas un aprendizaje artístico, tal como lo entendemos hoy y si estas contribuyen al desarrollo social de personas con capacidades diversas.

PALABRAS CLAVE: postmodernidad, art brut, aprendizaje artístico, locura, creatividad, arteterapia, discapacidad.

\section{ABSTRACT}

During the past 60 years, the art scene has undergone more changes than in any other time in history. The vanguards of Modernism left a clear testimony of the break with previous art, both in form and in content. At the end of Modernism one could still speak of disciplines including painting, sculpture, printmaking and drawing in this respect, but for the last 60 years other practices such as, installation, performance,

\footnotetext{
${ }^{1}$ Cecilia Mateo Sánchez: Universidad Católica San Antonio de Murcia, Departamento de Ciencias Sociales, Jurídicas y de la Empresa. Licenciada en Historia del Arte. Graduada en C.A.P. Postgraduada en Museología Contemporánea. Master universitario en Educación y Nuevas Tecnologías.
} 
body art, land art, video art or art brut, etc., which have been introduced to the field, however these can be included in the major genres of the arts known to all. The many innovations that have taken place in the art field since the Second World War forces us therefore, to call this new period differently from Modernism: postmodernism. It is in this context that we place raw art, art brut, marginal art or outsider art and the independence that these practices now have is what is meant by artistic learning. The need for new criticism and new historiography have arisen to understand, analyze and judge these new forms and mechanisms of production that lead to the understanding of postmodern art . The question now is whether behind these art forms there is artistic learning, as we understand it today, and if they contribute to the social development of people with diverse abilities.

KEY WORDS: postmodernism, art brut, artistic learning, madness, creativity, art therapy, disability.

\section{Cómo citar el artículo:}

Mateo Sánchez, C. y Mengual Luna I. (2014). El silencio del art brut: una visión diferente de un arte conectada a la vida. Revista de Ciencias de la Comunicación e Información, 19, 13-21.

http://doi.org/10.35742/revistaccomunicacioneinformacion.2014.19.13-21

"La locura es una respuesta creativa a un mundo insoportable" Yayoi Kusuma.

\section{INTRODUCCIÓN}

Si hacemos un recorrido por los principales teóricos del art brut como es el caso de Jean Dubuffet pintor y escultor francés, es de especial relevancia encontrarnos con un interés por un arte en el que no hay una base de aprendizaje artístico como tal, sino que surge de la necesidad de expresar las propias experiencias y el estado mental de las personas que lo generan. Las obras de estos enfermos o/y marginados mostraban la salud mental de una manera pura, no siendo influenciados por determinadas técnicas adquiridas de manera oficial o academicista.

Fueron autores desconocidos en su época porque su arte no fue catalogado como tal, quedando sus obras en las cárceles, asilos, geriátricos y psiquiátricos en donde ellos residían.

Sin embargo, Jean Dubuffet tras su estancia en el Sahara y tras haberse empapado de un arte denominado primitivo se interesó enormemente por este tipo de arte generado fuera de los circuitos oficiales. Este teórico, se dedicó al ataque del concepto tradicional de belleza, creando en 1948 la Compañía de Arte Bruto en Paris (Casado, 2006).

Podemos ver con este recorrido del arte bruto que ya en aquel tiempo, se mostraba el arte como un recurso de expresión emocional, aspecto que podemos llevarlo hoy día al arte como terapia. 


\section{OBJETIVOS}

Revisar los principales teóricos y su recorrido por el arte marginal para comprobar que el aprendizaje artístico sujeto, ligado y adquirido mediante el paso por academias y talleres no está presente en artistas con enfermedad mental y/o marginados. Es decir, el arte se puede producir sin el aprendizaje de éste contribuyendo al desarrollo personal de este colectivo en riesgo de exclusión social.

\section{METODOLOGÍA}

Para la elaboración de este artículo de tipo ensayo se ha realizado una revisión bibliográfica que ha encuadrado los principales artistas del arte marginal de la postmodernidad. Se utilizaron las bases de datos: Dialnet, Google Académico, Scientific Electronic Library Online (SciELO) y Tesis Doctorales en Red (TDR). Se emplearon los siguientes términos de búsqueda: postmodernidad, art brut, aprendizaje artístico, locura, creatividad, arteterapia, discapacidad. Igualmente se utilizaron traducidos al inglés: Postmodernism, art brut, artistic learning, madness, creativity, art therapy, disability. De los documentos resultantes de la búsqueda se seleccionaron aquellos más relevantes que informasen acerca de aspectos artísticos, creativos, históricos relacionados con la locura, la discapacidad y la creatividad en el arte bruto. Los criterios de relevancia que se utilizaron para su selección fueron: concordancia del contenido con los términos de búsqueda, número de citas recibidas, significación de los autores, idioma, criterio de relevancia aplicado por la misma base de datos.

\section{RESULTADOS}

Antes de que el termino art brut fuese acuñado, nos encontramos con artistas de referencia que pueden considerarse artistas brutos (Cabré, 2013). Han sido permanentes a lo largo de la historia, los artistas que han expresado a través de sus obras sus desequilibrados estados mentales. Las producciones de éstos nos han facilitado el conocimiento de sus patologías y /o enfermedades.

Mostramos brevemente alguno de ellos:

- Vincent Van-Gogh (1853-1890). En torno al año 1876 el autor citado abandona la enseñanza académica para crear de una forma completamente espontánea, en la cual va expresando progresivamente sus sentimientos y su estado mental. Se suicida de un disparo en 1890.

- Edvard Munch (1863-1944). Sufrió muy joven la pérdida de sus familiares y el espectro de la muerte, que llenó su niñez, lo acompañó durante toda su vida, convirtiéndose en una constante de sus obras. En 1908 sus problemas nerviosos, agravados por su alcoholismo, le obligan a ingresar en una clínica Psiquiátrica de Copenhague.

- Ernst Ludwig Kirchner (1880-1938). Líder del expresionismo alemán en 1915 sufrió una grave crisis física y psíquica como consecuencia de la guerra. Utiliza el arte como vehículo para mostrar su angustia y su obsesión. 
- Louis Wain (1860-1939) diagnosticado a los 57 años de esquizofrenia y autismo. Wain empezó a dibujar felinos, concretamente gatos para entretener a su esposa enferma. A medida que avanza la enfermedad de esta, los gatos de Wain, dejaron de ser gatos alegres que jugaban al golf para convertirse en criaturas inquietantes de mirada salvaje y colores tan fuertes como arrebatadores. En 1924, ingresa en una institución mental.

- Salvador Dalí (1904- 1989. Marcado por la muerte de su hermano declara en su autobiografía (Dalí, 1942) ser una segunda edición de su hermano muerto. Este artista encuentra su grandeza a través de la expresión de sus sentimientos. La muerte de su madre fue un acontecimiento que no logró comprender y que le encerró en una profunda depresión. Se definió a sí mismo como "perverso polimorfo, rezagado y anarquizante", "blando, débil y repulsivo". Padeció esquizofrenia paranoide.

- Yayoi Kusuma (1929). Artista japonesa de familia acomodada, creció aterrorizada y traumatizada por la experiencia a la que la sometía su madre, observar a su padre manteniendo relaciones con geishas que luego le obligaba a relatarle detalladamente. La artista afirma que, a partir de esas experiencias comenzó a sufrir alucinaciones visuales y auditivas, tendencias obsesivas y depresión, entre otras patologías mentales.

El máximo exponente de este alejamiento, de esa separación que las artes van adquiriendo del llamado arte academicista sujeto y marcado por los dictámenes de la sociedad, lo encontramos en la figura de Marcel Duchamp (1887-1968) artista francés que paso rápidamente por todas las corrientes artísticas en boga sin sentirse identificado con ninguna de ella. Su afán de experimentación fue una constante presente a lo largo de toda su trayectoria. En 1912 presentó su Desnudo bajando una escalera, $n$. ำ, obra en la que combinó elementos cubistas, futuristas y sus propias inquietudes sobre la representación del movimiento. La pintura fue rechazada por el Salón de los Independientes pero un año más tarde fue expuesta, en el Armory Show de Nueva York.

Este mismo año en 1913 inició los estudios previos para la obra El gran vidrio, una especie de simbiosis entre pintura y escultura.

Durante esa misma época realizó sus primeros ready-made, esto es, objetos cotidianos sometidos a escasa y en ocasiones sin alteración material, elevados a la categoría de arte por la mera voluntad del artista. Por ejemplo, un orinal firmado con seudónimo que tituló Fuente, constituye uno de los iconos más significativos del arte del siglo XX.

En la década de los 50 el director del Museo Solomon Guggenheim, James Johnson acuñó el término "tastebreakers" para referirse a individuos que abrían y ampliaban, las fronteras artísticas de su tiempo (Cabré, 2013).

Dos años más tarde, el crítico de arte francés Tapié (1952) centró sus estudios en los acontecimientos artísticos desarrollados tras la Segunda Guerra Mundial, acuñando otro término, art autre, para referirse a "arte otro" o informalismo, 
movimiento que abarca tanto las tendencias abstractas como las gestuales (action painting), que se desarrollaron en Francia y en el resto de Europa, tras el fin de la Segunda Guerra Mundial. Simultáneamente con el expresionismo abstracto estadounidense y dentro del informalismo encontramos corrientes tales como la abstracción lírica, la pintura materia, la nueva escuela de Paris, el tachismo o el art brut. Este arte informalista mostró una verdadera preocupación por el abandono de la forma ya fuese geométrica, naturalística o figurativa, centrándose en la espontaneidad y la irracionalidad.

No podemos hablar sobre arte bruto sin a conocer la figura de Jean Dubuffet, este pintor y escultor francés de la segunda mitad del siglo XX, fue el artista que acuñó dicha denominación. Fue uno de los primeros en reconocer e impulsar de una manera activa un arte que se encontraba fuera del sistema y de los círculos artísticos establecidos. El arte, defendía Dubuffet, no puede estar sujeto a nada que oscurezca o altere la expresión de las emociones. Abandonó la academia para estudiar de forma totalmente independiente, interesándose por profesionales que trabajaron fuera de la norma estética, un arte libre, de proposiciones intelectuales. Se embarcó en la aventura de descubrir la estética de lo extraordinario y de lo extraño, huyendo del reconocimiento de la cultura oficial. En 1945 comenzó a interesarse por el arte producido por los enfermos mentales, después de una larga estancia en Sahara como ya hemos comentado y de haber conocido y contactado con el arte primitivo.

\section{Declara Dubuffet (1992):}

Me encanta la locura; estoy muy enamorado de la locura. Siento la necesidad de que una obra de arte se cargue de sorpresa, que asuma un aspecto nunca visto, que desoriente mucho y transporte en el ámbito absolutamente imprevisto. Para mí, en cuanto el arte pierde ese carácter de distanciamiento, pierde esa eficacia; no sirve para nada.

Cardinal (1972) continuando en la misma línea de Dubuffet, denomina al arte creado por niños, presos, inmigrantes y loco- asilos como "arte marginal", refiriéndose al que genera producciones de carácter totalmente espontáneo, fuertemente imaginativas y que nos atraen por su pureza, al margen de cualquier convencionalismo desarrollando su labor creativa sin contacto alguno con las instituciones artísticas establecidas,.. Respondiendo a una fuerte motivación intrínseca y haciendo uso de materiales y técnicas inéditos. Una gran parte del arte marginal refleja estados mentales extremos, idiosincrasias particulares y/o elaborados mundos de fantasía.

El art brut, es una forma de crear en la cual la espontaneidad y el instinto prevalecen sobre la razón y la formación técnica o académica. Denominamos pues el arte bruto al generado fuera de los límites de la cultura oficial.

\subsection{Aprendizaje artístico en el art brut}

Eisner (1995) entiende que el aprendizaje artístico "no es un aprendizaje en una sola dirección. Aborda el desarrollo de las capacidades necesarias para crear formas 
artísticas, el desarrollo de capacidades para la percepción estética y la capacidad de comprender el arte como fenómeno cultural' (p. 59).

¿Dónde situamos el proceso del aprendizaje artístico en el art brut?

Durante toda la Historia del artes, los artistas, especialmente en la pintura y en escultura se han dedicado a trabajar copiando lo que veían en la naturaleza, en su realidad, eran artistas que trabajaban aprendiendo a trasladar a sus cuadros 0 esculturas la realidad que sus ojos contemplaban, cuando más se parecieran sus obras al objeto copiado, mejor consideración adquirían. Mimesis, es un concepto estético de la época de Aristóteles, imitación como fin principal del arte.

La copia y el parecido con la realidad copiada lo inundan todo. El arte, y la producción artística, tienen básicamente una función imitativa. En los artistas brutos, encontramos tanto los que copian obras de artistas conocidos, como los que producen sin ningún tipo de referente, en ambas producciones no encontramos un aprendizaje artístico como tal.

Dubuffet declara en 1992,

Con este término, art brut, designamos las obras realizadas por personas ajenas a la cultura artística, en las que el mimetismo, contrariamente de lo que les sucede a los intelectuales, está poco o nada presente, de tal modo que los autores recurren, en lo que se refiere a temas y materiales... a su interior y no a las vulgaridades del arte clásico o a la moda.

Un movimiento pictórico que no podemos dejar de conocer para entender estas nuevas concepciones es el Automatismo (Kuspit, 2003), estrategia creativa practicada principalmente por A. Masson. Parte del hecho de que se puede crear, de y a partir de todo, por tanto todos los medios de expresión son válidos. Se trata pues de dejar al inconsciente salir a la superficie y mostrar el interior del individuo.

Volvamos a Dubuffet, a raíz de la gran cantidad de escritos y reflexiones que publicó podemos extraer dos ideas fundamentales relacionadas con el aprendizaje artístico. La primera de ellas, es que cualquier persona puede crear o generar arte, con el aprendizaje solo de alguna habilidad técnica básica e incluso sin ella, de esta manera nos desprendemos del mimetismo o la copia.

La segunda, es que la primeras obras de los hombres primitivos, son una auténtica lección de creatividad, no se encuentra en ellos, ningún condicionante academicista, tampoco ningún aprendizaje, siendo todas ellas, producto de la libertad y la espontaneidad.

\subsection{Arte y terapia}

Al hablar de arte como expresión de las emociones tenemos que hablar necesariamente de arteterapia. 
Según Dalley y Case (1992), tenemos que recurrir al Neoclasicismo del siglo XIX para comenzar a hablar de los precursores, en él existía la creencia de que el estado mental de una persona podía leerse en sus representaciones o ilustraciones.

Durante el Romanticismo, hubo una concepción positiva del valor artístico y la imaginación, relacionada con la creencia de las capacidades naturales de la sanación del arte.

Las vanguardias artísticas: expresionismo, dadaísmo y surrealismo vuelven a valorar la espontaneidad y el arte de culturas primitivas, el arte infantil y el arte de enfermos mentales.

Entre 1930 y 1950, el trabajo artístico elaborado por personas con psicosis aparecía como una necesidad general para entender asuntos con mayor profundidad.

Con este panorama y acercándonos a la actualidad, en EE.UU la arteterapia ,surgió en la década de los 30 por medio de Margaret Naumburg, Edith Kramer y Florence Cane. Hay que destacar que Naumburg fue una de las primeras personas que empleó el término y el ejercicio de la arteterapia como profesión.

En Inglaterra durante la Segunda Guerra Mundial, el artista Adrian Hill al encontrarse convaleciente en un hospital se dedicó a pintar, actividad que contribuye y ayuda en su recuperación. Colaboró para que el arte fuera utilizado para ayudar a los soldados traumatizados por las experiencias de la guerra. Se le reconoce como el que aparentemente acuñó el término de arteterapia.

Arthur Segal intentó forjar lazos entre médicos y artistas, siendo consciente que ambos podían colaborar.

Otro pionero Edward Adamson trabajó en el Hospital psiquiátrico en Surrey durante la Segunda Guerra Mundial. En éste estableció un estudio de pintura.

Hans Prinzhorn (1886-1933). De origen alemán, estudió Historia del Arte y Filosofía. Más tarde recibió entrenamiento en la Medicina y la Psiquiatría. En 1919 se convirtió en asistente de Karl Wilmanns en el hospital psiquiátrico de la Universidad de Heidelberg. Amplió una colección de arte creada por enfermos mentales e iniciada por Emil Kraepelin. En 1921 la colección de Prinzhor contaba con más de 5000 obras. En 1922 publicó su primer y más importante libro "Actividad plástica de los enfermos mentales. Una contribución a la psicología y psicopatología de la configuración formal". En el que detalla la frontera entre la psiquiatría y el arte, la enfermedad y la auto-expresión. El estudio representa uno de los primeros intentos de analizar el trabajo generado de enfermos mentales, siendo reconocido como una de la figuras pioneras y más importante en los ámbitos de la psiquiatría y del arte.

Actualmente definimos arteterapia como disciplina o una técnica que usa el arte y los medios visuales como principal vía de comunicación en personas que por su enfermedad o disfunción, presentan dificultades para expresar sus conflictos de una manera verbal. Se desarrolla en contextos tales como hospitales, cárceles, centros 
de salud mental o escuelas. Reconocida por el ministerio de salud y como profesión en EE.UU, Canadá, Inglaterra o Israel. Cada vez son más los países que han ido introduciendo la profesión de Arteterapia en la enseñanza pública de forma regulada. Algunos de estos son Australia, Canadá, Estados Unidos, España, Holanda, Inglaterra e Israel.

Actualmente, son muchas las asociaciones y organizaciones de arteterapia que se han ido constituyendo alrededor del mundo.

Sus objetivos principales son expresar sentimientos difíciles o imposibles de hablar y aumentar la motivación y la autoestima del paciente mentalmente enfermo.

La Asociación Británica de Arteterapia (BAAT) (Citado en Ruddy y Milnes, 2008) la describe como un tipo de psicoterapia donde el arte es el principal modo de comunicación. Siendo su ámbito de diagnóstico muy amplio, desde problemas emocionales a situaciones neurológicas. Hay que destacar que no es necesario que el cliente conozca o tenga experiencia previa en el arte.

\section{CONCLUSIONES Y DISCUSIÓN}

Mediante esta revisión hemos conocido una serie de artistas que o no están ligados a procesos de enseñanza-aprendizaje oficiales, o si lo han estado los han abandonado rápidamente. Se ha contrapuesto un arte cultural, frente a un arte no cultural, se le ha dado prioridad a un arte no asfixiado de estereotipos, estos artistas, han creado y se han expresado de una manera totalmente independiente, desligados de la academia y de la oficialidad y generando de una forma completamente espontánea, han buscado una nueva pureza en el arte.

Hemos visto como el aprendizaje artístico no tiene necesariamente que ser el conductor ni tiene porque haber sido adquirido para el desarrollo de capacidades artísticas de personas con enfermedad mental, se puede producir sin el conocimiento de éste. Lo que se confirma es la existencia, cada vez más arraigada, de disciplinas alternativas, como es la arteterapia que, aunque no es un tratamiento farmacológico, contribuyen a fortalecer el desarrollo personal, la autoestima y motivación de personas con enfermedad mental, discapacidad intelectual y en riesgo de exclusión social.

\section{REFERENCIAS}

Cabre, V. (2013). Jean Dubuffet. Temas de Psicoanalisis, 5, 1-9.

Cardinal, R. (1973). Art and mental illness. Nueva York: Praeger Publishers.

Casado, M. (2006). A propósito del arte marginal y sus límites. Paperback, 2, 1-8.

Dali, S. (1942). The secret life of Salvador Dalí. Nueva York: The Dial Press.

Dalley, T. \& Case, C (1992). The Handbook of Art Therapy. Londres: Tavistock/Routledge. 
Dubuffet, J. (1992). El hombre de la calle ante la obra de arte. Madrid: Debate.

Eisner, E. (1995). Educar la visión artística. Barcelona: Paidós Ibérica.

Kuspit, D. (2003). Signos de la Psique en el arte Moderno y Postmoderno. Madrid: Akal.

Romero, J. (2006) Creatividad, arte, locura: una red de conceptos limítrofes. Revista Arte, Individuo y Sociedad, 18, 131-141.

Ruddy, R. y Milnes, D. (2009). Art therapy for schizophrenia or schizophrenia-like illnesses. The Cochrane Library, 3.

Tapié, M. (1952). Un art autre. Paris: Gabriel-Giraud. 